\title{
From "open" to robotic assisted thoracic surgery: why RATS and not VATS?
}

\author{
Sara Ricciardi ${ }^{1 \#}$, Federico Davini $^{2 \#}$, Carmelina Cristina Zirafa $^{2}$, Franca Melf $^{2}$ \\ ${ }^{1}$ Unit of Thoracic Surgery, ${ }^{2}$ Unit of Minimally Invasive and Robotic Thoracic Surgery, Robotic Multispeciality Center for Surgery, University \\ Hospital of Pisa, Italy \\ \#These authors contributed equally to this work. \\ Correspondence to: Sara Ricciardi. Via Paradisa 2, 56124 Pisa, Italy. Email: ricciardi.sara87@gmail.com.
}

Received: 19 April 2018; Accepted: 26 April 2018; Published: 22 May 2018.

doi: 10.21037 /jovs.2018.05.07

View this article at: http://dx.doi.org/10.21037/jovs.2018.05.07

Since 1990's, when the first video-assisted thoracoscopic surgery (VATS) lobectomy was performed, the interest in minimally invasive surgery (MIS) to treat lung cancer has grown widely (1).

Less post-operative pain, improved cosmetic result, shorter hospitalization, lower morbidity and perioperative mortality are just some of the remarkable benefits of MIS when compared with open surgery. Furthermore, several studies have demonstrated that minimally invasive technique is not inferior in terms of oncologic outcomes also in advanced stages lung cancer (2).

Throughout the last decades, several different types of minimally invasive approaches have been developed, such as the robot assisted thoracic surgery (RATS) or uniportal VATS (U-VATS), and surgeons are still debating on which is the most advantageous procedure to perform a MIS lobectomy.

Whilst VATS technique supporters claim that robotic surgery is expensive and only for elitist hospitals, the technological evolution and provided benefits of RATS, both for patients and for surgeons, are unquestionable.

The robotic system guarantees a much-improved vision thanks to the 10 times magnification and the depth perception of its $3 \mathrm{D}$ high definition camera, which also provides a stable view eliminating the movements of the assistant's arm. In addition, the robotic instruments with seven degrees of freedom (7DOF) allow to perform complex surgical maneuvers safely, reaching comfortably narrow spaces. Therefore, the vessels and the lymph nodes dissection are more accurate and meticulous when compared with VATS technique $(3,4)$.

Lymphadenectomy is one of the challenging step during
VATS lobectomy due to the absence of articulations and the technical difficulties, such as instrument impingement within the slender mediastinal space.

Conversely, the wristed robotic instruments make the lymphadenectomy an effortless procedure.

Furthermore, despite it is still debated, several studies have demonstrated the superiority of robotic lymphadenectomy compared with VATS. Dissonant data is found on the radicality of nodal yield during VATS procedures, commonly a lower median number of harvested lymph nodes with a fewer nodal upstaging, particularly for the N2 stations, are reported in VATS procedures when compared to open surgery $(5,6)$.

On the other hand, numerous studies have confirmed the equivalence between robotic nodal dissection and open nodal dissection. In fact, the median number of lymph nodes resected through robotic approach appears to be the same of open surgery $(7,8)$. Moreover, thanks to the precision of the robotic instruments, nodes can be harvested as a whole without breaking the capsule.

When considering the rate of nodal upstaging as an indicator of the quality of surgery, several studies have exposed that RATS is able to ensure a comparable, if not superior, upstaging to the one achieved with thoracotomy, suggesting that robotic lobectomy guarantees an equivalent oncological radicality to open surgery $(7,9,10)$.

In order to have more precise and accurate movements, the robotic platform is also equipped with tremor filtering (6-Hz motion filter) and motion scaling systems that counteract physiologic hands tremor, allowing to obtain the finest surgical movements. This seems to guarantee a better dissection, with a reduction in blood loss, and to perform 
more accurate sutures (11). Thoracoscopic instruments, with their long shafts, amplify the unintentional actions and force inverted movements along obligate directions. Moreover, during VATS procedure the surgeon inevitably has to maintain an uncomfortable standing position, sometimes with mirrored vision, particularly exhausting during difficult and long operations. Whereas the robotic system allows the surgeon to sit in ergonomic position and to perform any movement in a comfortable way, thus reducing fatigue and muscular struggle. In addition, the surgeon can also freeze a robotic instrument in a desired position, which is particularly useful to easily retract the lung obtaining an optimal exposition of structures. In case of complication, such as an uncontrollable bleeding, the surgeon can safely leave the robotic clamp locked on the vessel and, with both hands unrestrained, troubleshoot the drawback.

To aid surgeon during challenging procedures, the robotic platform is equipped with Firefly (fluorescence capable vision system) which offers integrated high-resolution nearinfrared fluorescence image in 3-dimensional vision, taking advantage of this technology to identify vascular anatomy, lymph node mapping and real-time pinpoint of lesions.

Regarding the learning curve, thanks to the intuitive robotic movements, 20 RATS lobectomies appear to be sufficient for a skilled thoracic surgeon, in contrast to VATS $(12,13)$. The robotic system is also provided with simulator and with a dual console, which help the trainees to become skilled surgeons; their application is part of a wellestablished pathway which implies teaching and proctoring during the early phases of the robotic experience.

It has been demonstrated that in high volume centres the outcome improves significantly applying the standardization of the technique (14). Thanks to the evolution of the technology in parallel to the improvement of the technique, the indication for robotic procedures has been widely extended. From its initial early stage non-small cell lung cancer (NSCLC) treatment, the use of robotic platform has now been extended to more complex procedures such as sleeve lobectomy or locally advanced lung cancer, with outstanding results $(15,16)$.

The most contested side of RATS is the high expenses involved. However, it has been demonstrated that in high volume specialised hospitals a strategy based on high volume cases and on the standardization of the technique, allows the sustainability of this advanced technology (17).

Nowadays the da Vinci surgical system (Intuitive Surgical Inc., Sunnyvale, CA, USA) is the only complete surgical platform worldwide available, but arguably new robotic systems will be available in the future. The introduction of new platforms on the market is bound to create a competition between the companies that will decrease the average costs of this surgical technique.

Moreover, a prospective future system could create a hybrid operating room in which the surgeon could interact with the patient's preoperative exams, planning the best surgical approach with personalized steps to be taken for each case. Thanks to the possibility to develop a 3D truthful anatomical model, the operator can also practice before the intervention in order to acquire the necessary confidence to proceed faster and in a more accurate way, also in complex cases.

The unquestionable benefits and the continuous upgrade of robotic system allow to put the highest available technology in the hand of the surgeon to perform a broad range of different procedure in a safe, effective and ergonomic way.

The advantages of RATS, both for patient and for the surgeon, suggest the superiority of this MIS technique. In fact, even though in its infancy the robotic system has limitless potentiality and increasing applications persuading us that this is the best minimally invasive procedure in thoracic surgery.

\section{Acknowledgements}

We thank Teresa Hung Key for linguistic accuracy checking.

\section{Footnote}

Conflicts of Interest: The authors have no conflicts of interest to declare.

\section{References}

1. Roviaro G, Rebuffat C, Varoli F, et al. Videoendoscopic pulmonary lobectomy for cancer. Surg Laparosc Endosc 1992;2:244-7.

2. Sihoe AD. Reasons not to perform uniportal VATS lobectomy. J Thorac Dis 2016;8:S333-43.

3. Yang S, Guo W, Chen X, et al. Early outcomes of robotic versus uniportal video-assisted thoracic surgery for lung cancer: a propensity score-matched study. Eur J Cardiothorac Surg 2017. [Epub ahead of print].

4. Oh DS, Reddy RM, Gorrepati ML, et al. Robotic-Assisted, Video-Assisted Thoracoscopic and Open Lobectomy: 
Propensity-Matched Analysis of Recent Premier Data. Ann Thorac Surg 2017;104:1733-40.

5. Merritt RE, Hoang CD, Shrager JB. Lymph node evaluation achieved by open lobectomy compared with thoracoscopic lobectomy for N0 lung cancer. Ann Thorac Surg 2013;96:1171-7.

6. D'Amico TA, Niland J, Mamet R, et al. Efficacy of mediastinal lymph node dissection during lobectomy for lung cancer by thoracoscopy and thoracotomy. Ann Thorac Surg 2011;92:226-31; discussion 231-2.

7. Cerfolio RJ, Bryant AS, Skylizard L, et al. Initial consecutive experience of completely portal robotic pulmonary resection with 4 arms. J Thorac Cardiovasc Surg 2011;142:740-6.

8. Ricciardi S, Cardillo G, Zirafa CC, et al. Robotic lobectomies: when and why? J Vis Surg 2017;3:112.

9. Toker A, Ozyurtkan MO, Demirhan O, et al. Lymph Node Dissection in Surgery for Lung Cancer: Comparison of Open vs. Video-Assisted vs. Robotic-Assisted Approaches. Ann Thorac Cardiovasc Surg 2016;22:284-90.

10. Velez-Cubian FO, Ng EP, Fontaine JP, et al. RoboticAssisted Videothoracoscopic Surgery of the Lung. Cancer Control 2015;22:314-25.

11. Farivar AS, Cerfolio RJ, Vallieres E, et al. Comparing

doi: 10.21037/jovs.2018.05.07

Cite this article as: Ricciardi S, Davini F, Zirafa CC, Melfi F. From "open" to robotic assisted thoracic surgery: why RATS and not VATS? J Vis Surg 2018;4:107. robotic lung resection with thoracotomy and videoassisted thoracoscopic surgery cases entered into the Society of Thoracic Surgeons database. Innovations (Phila) 2014;9:10-5.

12. Melfi FM, Mussi A. Robotically assisted lobectomy: learning curve and complications. Thorac Surg Clin 2008;18:289-95, vi-vii.

13. Cheufou DH, Mardanzai K, Ploenes T, et al. Effectiveness of Robotic Lobectomy-Outcome and Learning Curve in a High Volume Center. Thorac Cardiovasc Surg 2018.

14. Tchouta LN, Park HS, Boffa DJ, et al. Hospital Volume and Outcomes of Robot-Assisted Lobectomies. Chest 2017;151:329-39.

15. Schmid T, Augustin F, Kainz G, et al. Hybrid videoassisted thoracic surgery-robotic minimally invasive right upper lobe sleeve lobectomy. Ann Thorac Surg 2011;91:1961-5.

16. Veronesi G, Novellis P, Difrancesco O, et al. Robotic assisted lobectomy for locally advanced lung cancer. J Vis Surg 2017;3:78.

17. Veronesi G, Cerfolio R, Cingolani R, et al. Report on First International Workshop on Robotic Surgery in Thoracic Oncology. Front Oncol 2016;6:214. 